\title{
Synthesis and Characterization of Carbon Nanofibers Grown on Powdered Activated Carbon
}

\author{
Yehya M. Ahmed, ${ }^{1}$ Abdullah Al-Mamun, ${ }^{2}$ Ahmad T. Jameel, ${ }^{2}$ Ma'an Fahmi R. AlKhatib, ${ }^{2}$ \\ Mutiu K. Amosa, ${ }^{3}$ and Mohammed A. AlSaadi ${ }^{4}$ \\ ${ }^{1}$ Department of Civil Engineering, Faculty of Architecture and Built Environment, Limkokwing University of Creative Technology, \\ 63000 Cyberjaya, Malaysia \\ ${ }^{2}$ Nanoscience and Nanotechnology Research Group (NANORG), Faculty of Engineering, \\ International Islamic University Malaysia (IIUM), Jalan Gombak, 53100 Kuala Lumpur, Malaysia \\ ${ }^{3}$ School of Chemical and Metallurgical Engineering, University of the Witwatersrand, 1 Jan Smuts Avenue, \\ Braamfontein 2000, Johannesburg, South Africa \\ ${ }^{4}$ Nanotechnology and Catalysts Research Center (NANOCAT), Department of Chemical Engineering, \\ Faculty of Engineering, University of Malaya, 50603 Kuala Lumpur, Malaysia
}

Correspondence should be addressed to Abdullah Al-Mamun; mamun@iium.edu.my

Received 7 June 2016; Accepted 30 October 2016

Academic Editor: Enkeleda Dervishi

Copyright (C) 2016 Yehya M. Ahmed et al. This is an open access article distributed under the Creative Commons Attribution License, which permits unrestricted use, distribution, and reproduction in any medium, provided the original work is properly cited.

Carbon nanofibers (CNFs) were synthesized through nickel ion $\left(\mathrm{Ni}^{2+}\right)$ impregnation of powdered activated carbon (PAC). Chemical Vapor Deposition (CVD) using acetylene gas, in the presence of hydrogen gas, was employed for the synthesis process. Various percentages $\left(1,3,5\right.$, and $7 \mathrm{wt}$. \%) of $\mathrm{Ni}^{2+}$ catalysts were used in the impregnation of $\mathrm{Ni}^{2+}$ into PAC. Field Emission Scanning Electron Microscope (FESEM), Fourier Transform Infrared (FTIR) Spectroscopy, Energy Dispersive X-Ray Analyzer (EDX), Transmission Electron Microscopy (TEM), Thermal Gravimetric Analysis (TGA), zeta potential, and Brunauer, Emmett, and Teller (BET) were utilized for the characterization of the novel composite, which possessed micro and nanodimensions. FESEM and TEM images revealed that the carbonaceous structure of the nanomaterials was fibrous instead of tubular with average width varying from 100 to 200 nanometers. The PAC surface area increased from $101 \mathrm{~m}^{2} / \mathrm{g}$ to $837 \mathrm{~m}^{2} / \mathrm{g}$ after the growth of CNF. TGA combustion temperature range was within $400^{\circ} \mathrm{C}$ and $570^{\circ} \mathrm{C}$, while the average zeta potential of the nanocomposite materials was $-24.9 \mathrm{mV}$, indicating its moderate dispersive nature in water.

\section{Introduction}

Activated carbon (AC) is an adsorbent used on an enormous scale in gaseous and aqueous purifications, metal extraction, and medicine, amongst other applications [1]. Several studies reported that $\mathrm{AC}$ is efficient in the sorption of various types of bioresistant pollutants from the environment [2]. Besides the use of AC for isolation of metals from aquatic environment by adsorption process which is a well-established process [3-5], activated carbons are also employed as catalyst supports for various applications $[6,7]$.

Carbon nanofibers (CNFs) are hydrophobic, generally, without significant functional groups [8], and the CNFs have recently received added attention due to their potential usages in various research and industrial sectors. For instance, chemical industry, material science, and environmental and energy storage specialties [9]. Preparation of polymer composites carried out through the dispersion of CNF into the polymer matrices is also reported [10], while CNF utility in the adsorption of volatile organic compounds (VOC) such as alkanes, aromatics and chloro-hydrocarbons was established in the reports of previous investigations [11]. Factually, CNFs are potential materials for various engineering applications due to their unique chemical, physical, mechanical, and electrical properties $[12,13]$. The diameter of a carbon nanofiber ranges between $50 \mathrm{~nm}$ and $200 \mathrm{~nm}$ and that of the carbon nanotubes 
is usually less than $100 \mathrm{~nm}$. Studies on the variation in diameter of fibrous graphitic materials are reported by Shaffer and Sandler [14].

Several methods have been used in the synthesis of CNF, such as the utility of various catalysts and carbon sources [15-20]. In laser ablation, a graphite target source with little amount of catalysts (usually a transition metal) is exposed to a laser beam which vaporizes the graphite and initiate the growth of CNF just in front of the target [16]. It is pertinent to note that in the CVD method, which is the most common method for bulk production of carbon nanomaterials, different hydrocarbons (carbon sources) are deposited on various metal catalysts (Fe, Co, and $\mathrm{Ni}$ ) at temperatures between 500 and $1200^{\circ} \mathrm{C}$ [21].

The process parameters that are important for the synthesis of CNF are the ratio of hydrogen to carbon source flow, reaction time, and temperature. The effect of hydrogen gas can be acceleration and suppression. Effect of acceleration on the formation of carbon may be interpreted in two ways. The first theory suggests decomposition of inactive metal carbides by hydrogen to form active metal [22]. The other explanation proposes that hydrogen removes surface carbon and precursors of carbon which leads to active site blocking [5]. The effect of suppressing has also been suggested to occur due to the surface hydrogenation reactions. The effect of hydrogen on the formation of CNF from the decomposition of metal catalyzed by hydrocarbon molecules is thought to convert inactive metal carbides into catalytically active metallic state which prohibits the formation of graphite layers on the surface of the metal particles. Moreover, the diffusion and precipitation rates of carbon atoms are affected by temperature and availability of carbon atoms [23]. The precipitation of carbon on the metal catalysts can be categorized into surface and internal. It is reported that, at low temperatures, carbon atoms can only precipitate on the surface areas of the catalyst particles, while the diffusing and dissolution rates of carbon atoms increase with the temperature, and carbon atoms can get into the internal areas of the catalysts to form CNF [24].

The growth time amongst other factors plays a major role in tailoring the morphology of nanomaterials in terms of diameter, dense of growth, and so forth. $[25,26]$. Two growth stages are reported in the literature. In the initial stage, a part of the nickel surface is covered by carbon leading to formation of relatively catalyst-rich carbon area. In the next stage dissolved carbon is released to form CNF with the lift-off of the former catalyst-rich carbon particles out of the substrate surface. Lastly, the decomposition of hydrocarbon on the new catalyst initiates new deposition of carbons from the same particle covering the host surface completely by a dense layer of CNF. The role of reaction time was previously studied on the growth of CNF from the decomposition of ethylene over $\mathrm{Ni} / Y$-zeolite [27]. The reaction duration was $60 \mathrm{~min}$ while a total time of $3 \mathrm{hr}$ was needed for the synthesis of CNF in the decomposition of ethylene over an unsupported $\mathrm{Ni}-\mathrm{Cu}$ alloy [28].

The main objective of this study was to use oil palm kernel shell based PACs as a solid substrate for the growth of CNF on nickel impregnated PACs by CVD method.
Determination of various physicochemical properties of the new nanomicrocomposite carbon material was done as well.

\section{Experimental}

The following materials and equipment were used for the synthesis and characterization processes.

2.1. Materials and Reagents. Palm kernel shell based granular activated carbon (GAC) was obtained from Effigen Carbon Sdn. Bhd., Malaysia. The GAC was bought in $20 \mathrm{~kg}$ bags and grinded to powdered activated carbon (PAC) of sizes ranging from 100 to $250 \mu \mathrm{m}$. Nickel (II) nitrate hexahydrate, 99\%, was purchased from Acros organics, USA, for the preparation of the nickel catalyst, which was impregnated into the PAC. Acetone QP was obtained from HmbG Chemicals, Germany, for the purpose of dissolving the nickel salt in readiness for the impregnation process. Purified nitrogen gas was purchased from Fuelink Marketing Sdn. Bhd., Selangor, Malaysia, which served as carrier gas for the calcination of the nickel catalyst growth of the CNF on PAC. Purified hydrogen gas was purchased from Malaysian Oxygen Bhd. and utilized for reduction of nickel catalyst. Purified acetylene gas from MOX-Linde Gases Sdn. Bhd. was used as a carbon source for the growth of CNF on PAC surface.

2.2. Equipment and Instrumentation. Philips grinder was used to crush the raw granular activated carbon to form PACs. Retsch sieve shakers model AS200 of German product, digit, and Max amplitude of 100 was used to sieve the grinded activated carbon to desired fraction size. A\&D four digits weighing balance (HR-202i, Japan) was used for all the weighing measurements. The weighing range of the balance is from 0.001 to $220 \mathrm{~g}$. Ultrasonic bath model (JAC 2010 P) from South Korea was used for the mixing of PAC and nickel solution to impregnate the metal onto the substrate. The bath is equipped with heater $\left(\max .70^{\circ} \mathrm{C}\right)$, timer for 99 seconds, and three sonication levels. Lenton, England triple stage horizontal tubular ceramic reactor (diameter, $50 \mathrm{~mm}$; length, $1500 \mathrm{~mm}$ ), was used for the CVD process for the growth of CNF. The maximum heating temperature for the furnace is $1200^{\circ} \mathrm{C}$. At the heating area, the ceramic tube utilized a resistance heating glass wool from Isolite Ceramic Fiber Sdn. Bhd. Malaysia. A drying oven model 600 from Memmert, Germany, of maximum temperature $220^{\circ} \mathrm{C}$ was used to dehumidify the samples of PAC and CNF-PAC.

FESEM model 6700F from JEOL Company (Japan) was used to determine the morphological features of the CNF. Energy Dispersive X-ray Analyzer was attached to the FESEM for the determination of structure of the PAC and CNF. High-resolution TEM model JEM-2010 from JEOL Company (Japan) was used to determine the diameter and length of the CNF. Automated Gas Sorption System (Quantachrome Autosorb) from Quantachrome Company (USA) was used to calculate the surface area of the powdered activated carbon before and after the CNF growth. FTIR model 100 from Perkin Elmer (USA) was used for the classification of the functional groups on the PAC, impregnated PAC, and CNF. 


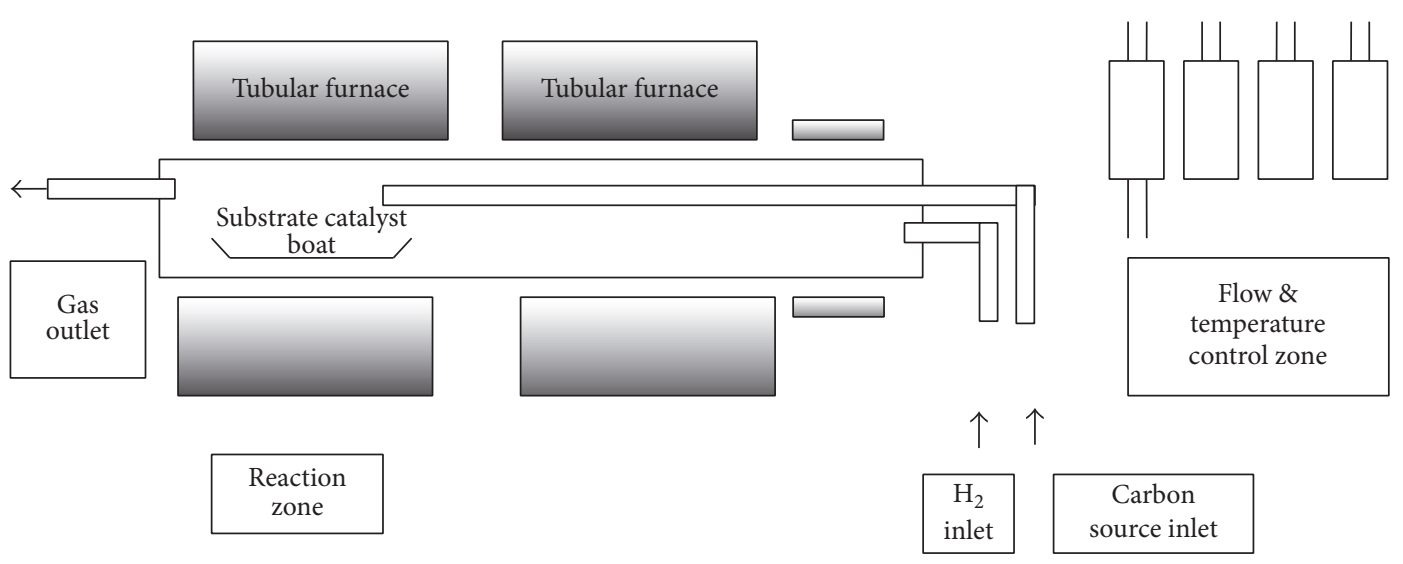

FIGURE 1: Schematic diagram of multipurpose triple stage CVD system.

Perkin Elmer Instruments Pyris diamond TG/DTA (USA) was used to study the thermogravimetric behavior of PAC and CNF-PAC.

2.3. Powdered Activated Carbon Samples Preparation. Palm kernel shell based granular activated carbon (GAC) was grinded and sieved. Powdered activated carbon (PAC) with particle size between 100 and $250 \mu \mathrm{m}$ was collected and kept in sealed plastic bags for production and adsorption studies.

2.4. Catalyst Preparation. The incipient wetness impregnation was used to impregnate nickel nitrate salt onto PAC by dissolving the nickel salt in acetone adopting method described elsewhere [25]. Four different weight percent (w/w\%) samples of nickel catalyst were prepared and the ratios were calculated based on the Ni to PAC in the sample. Using the ratios, $1,3,5$, and $7 \%$, preparations were made by mixing the required amount of nickel nitrate hexahydrate $\mathrm{Ni}\left(\mathrm{NO}_{3}\right)_{2} \cdot 6 \mathrm{H}_{2} \mathrm{O}$ with small amount of acetone (in universal glass bottle) until all the nickel salt was totally dissolved and homogenously mixed.

2.5. PAC Impregnation. The catalyst was mixed with specified amount of PAC. The mixture was then sonicated in the ultrasonic bath for $30 \mathrm{~min}$ at room temperature and with high sonication speed. The nickel impregnated PAC was left in the water bath at temperature of $56^{\circ} \mathrm{C}$ and sonicated for $12 \mathrm{~h}$ to ensure complete impregnation process and evaporation of any excess acetone.

2.6. CNF Growth. Catalyst substrate was fixed in a ceramic alumina boat in the tubular ceramic reactor (Figure 1). Calcination was carried out at $350^{\circ} \mathrm{C}$ with nitrogen gas flow rate of $200 \mathrm{~mL} / \mathrm{min}$ for $2 \mathrm{~h}$ into the $\mathrm{CVD}$ reactor under atmospheric pressure. The solid was then reduced by hydrogen gas at $400^{\circ} \mathrm{C}$ and $120 \mathrm{~mL} / \mathrm{min}$ flow rate for $2 \mathrm{hr}$. The reduced catalysts were, then, cooled down to ambient temperature and weighed to determine the amount of moisture present. The synthesis of CNF was carried out on the reduced catalysts by the CVD reactor at atmospheric pressure and in the
TABLE 1: Yield percentage of the CNF growth on impregnated PAC.

\begin{tabular}{lccc}
\hline $\begin{array}{l}\text { Catalyst } \\
\text { percentage } \\
(\%)\end{array}$ & $\begin{array}{c}\text { Weight }(\mathrm{g}) \text { of } \\
\text { sample before CNF } \\
\text { growth }\left(w_{0}\right)\end{array}$ & $\begin{array}{c}\text { Weight }(\mathrm{g}) \text { of } \\
\text { sample after CNF } \\
\text { growth }\left(w_{1}\right)\end{array}$ & Yield (\%) \\
\hline 1 & 1.2 & 1.56 & 30 \\
3 & 1.2 & 2.16 & 68 \\
5 & 1.3 & 2.02 & 55 \\
7 & 1.3 & 1.86 & 43 \\
\hline
\end{tabular}

presence of a $\mathrm{C}_{6} \mathrm{H}_{6} / \mathrm{H}_{2}$ mixture (total flow rate, 20 and $100 \mathrm{~mL} / \mathrm{min}$ ) at $650^{\circ} \mathrm{C}[25]$. The raw $\mathrm{CNF}$ was cooled down to ambient temperature followed by measurement of the product weight. The processes of calcination, reduction, and growth processes of CNF were done for the four different weight percent samples of the catalyst. The best samples in terms of the percentage yield were chosen for further studies. The yield was calculated from the difference between the weight of the sample after calcination and reduction were done (before CNF growth) and its weight after the growth of CNF completed.

\section{Results and Discussion}

3.1. Effect of Ni Catalyst on CNF Yield. After the impregnation, calcination, and reduction of the catalyst and the growth of the CNF, the product was weighed and the yield, $\%$, of the grown CNF was determined by using the formula (1), where $w_{0}$ and $w_{1}$ were the initial and final weights of the impregnated substrate (PACs). The results are shown in Table 1 .

$$
\% \text { Yield }=\frac{w_{1}-w_{0}}{w_{0}} \times 100
$$

The yield calculations (\%) showed that the highest yield of CNF was achieved from the 3\% catalyst and the process condition was used for further production of PAC-CNF samples. A percentage weight catalyst higher than $3 \%$ gave less percentage yield, and this is attributable to the agglomeration of $\mathrm{Ni}$, while $1 \%$ gave lesser yield when compared with that 


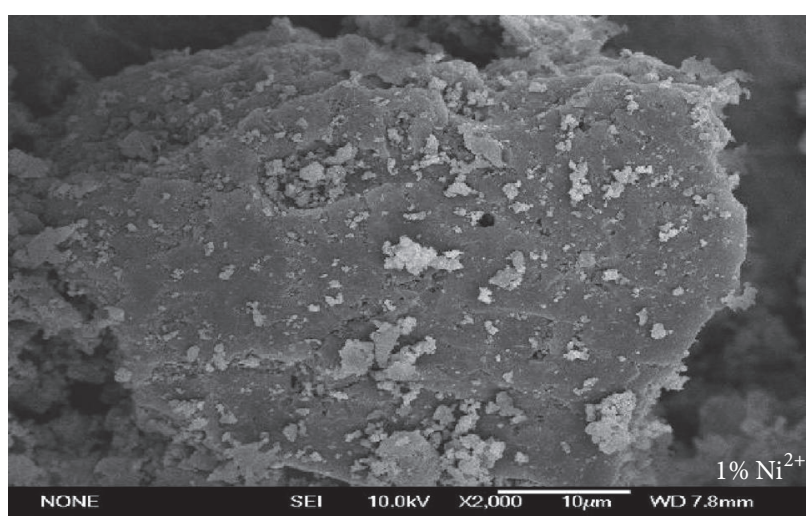

(a)

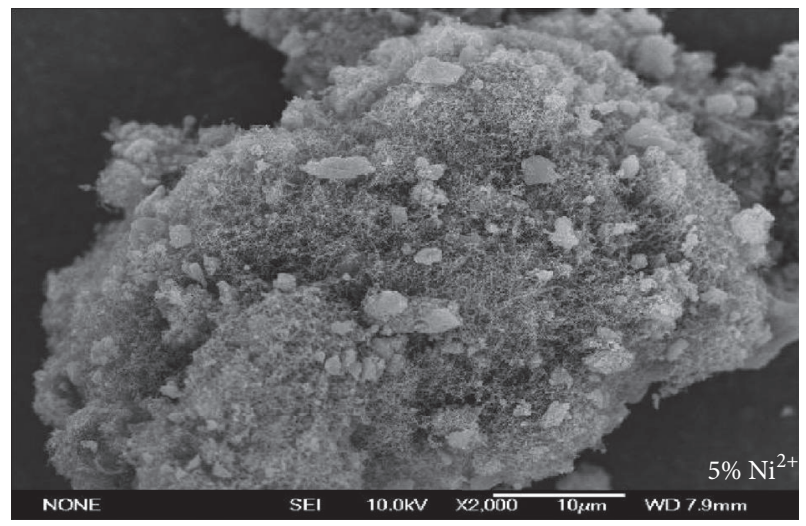

(c)

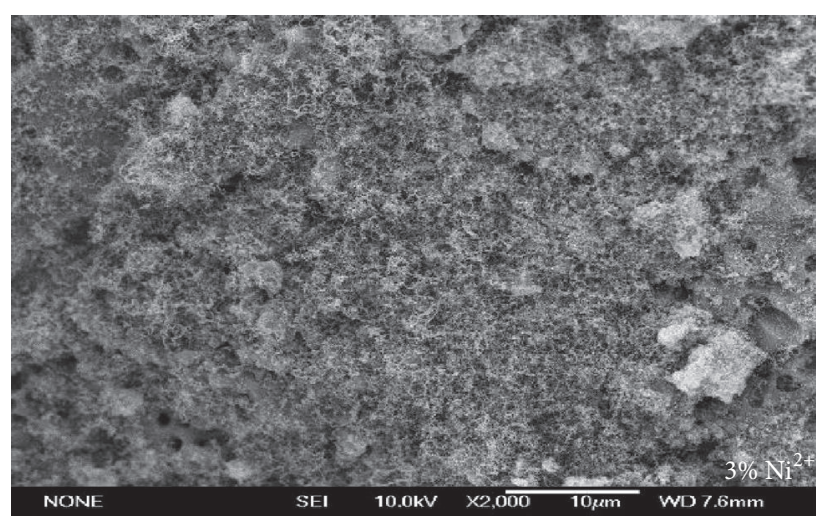

(b)

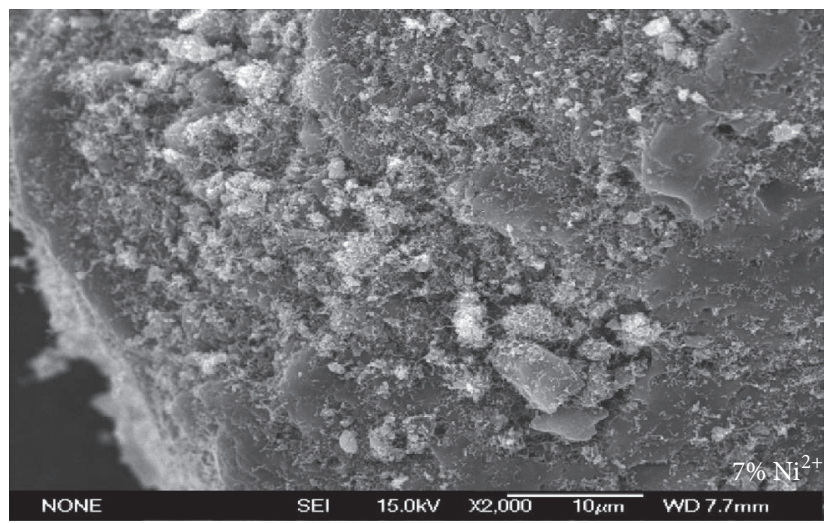

(d)

Figure 2: Field Emission Scanning Electron Microscope image of the CNF for (a) 1\% catalyst, (b) 3\% catalyst, (c) 5\% catalyst, and (d) $7 \%$ catalyst.

of $3 \%$. This effect may be attributed to the lesser amount of nickel exposed to the carbon as concluded in previously reported similar investigation [27]. The distribution of the CNF growth on the impregnated PAC is shown in the FESEM images for the various catalyst percentage ratios are shown in Figure 2. The poor growth shown in Figure 2(a) was due to the low weight percentage of nickel catalyst used for the impregnation [29].

The FESEM images showed that the catalyst of $3 \mathrm{wt} . \%$ gave the best growth of CNF and it matches with the yield calculations showed in Table 1 . Increase in the catalyst percent weight increased the chance of the growth as reported in earlier investigations [30], while further increase in the catalyst percentage led to the agglomeration of nickel particles and subsequently a decrease in the growth of CNF as depicted in Figure 2. This observation is in conformity with previous reports $[31,32]$.

3.2. FESEM Results. Field Emission Scanning Electron Microscopy (FESEM) technique was used for the analysis of physical morphology of the surface of the prepared samples. Figures 3(a), 3(b), and 3(c) showed the images of the raw material (PAC), impregnated PAC, and the prepared PAC-CNF samples with $2000 \mathrm{x}$ magnification, respectively. Figure 3(c) showed the whole surface of the substrate covered with the grown CNF, and compared with the images in Figures 3(a) and 3(b), the coverage with the grown CNF led to increase in the original substrate's adsorption properties. Figure 3(d), which represented the higher magnification of Figure 3(c), showed the diameters of the CNF ranging from the average of 100 to $160 \mathrm{~nm}$ with the presence of many white confirmed to be Ni particles by the EDX analysis.

3.3. TEM Results. The Transmission Electron Microscopy (TEM) analysis proved the formation of the as-prepared CNF. Figures 4(a) and 4(b) show that the fiber has an average diameter range between 100 and $200 \mathrm{~nm}$ as matched with images of FESEM above. Closely related observations were made in the previous TEM studies for the confirmation of CNF dimensions [33-35]. The images also revealed the firmness of the amorphous carbon structure. The high quality image (Figure 5) showed the layers by layers CNF structure as indicated by the white arrows. The image showed that the CNF microstructures possessed defective platelets and more graphitic platelets that was similar to previous reports [3638].

3.4. Surface Area of the PAC-CNF. Using computermonitoring system, the adsorbed nitrogen gas volume, equilibrium pressures, and BET surface area of the sample 


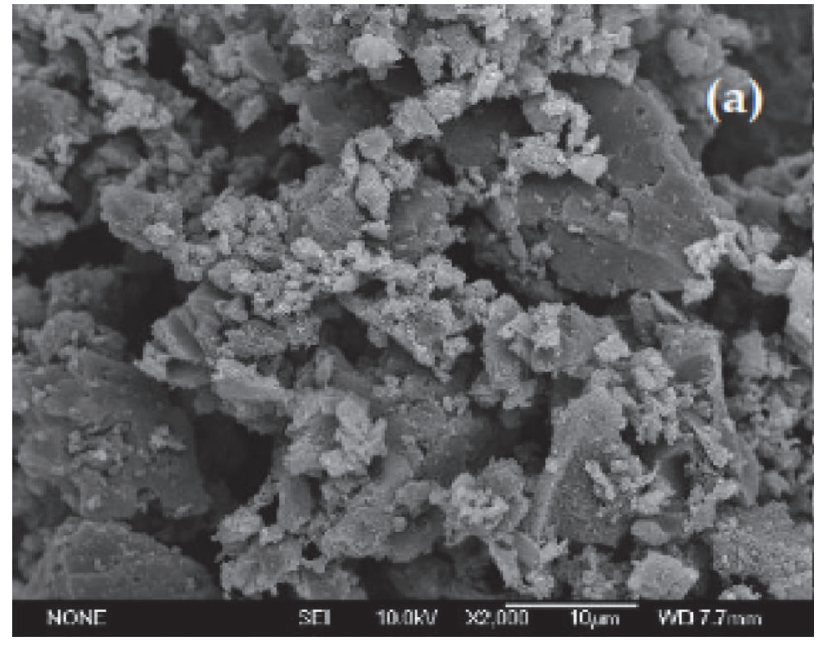

(a)

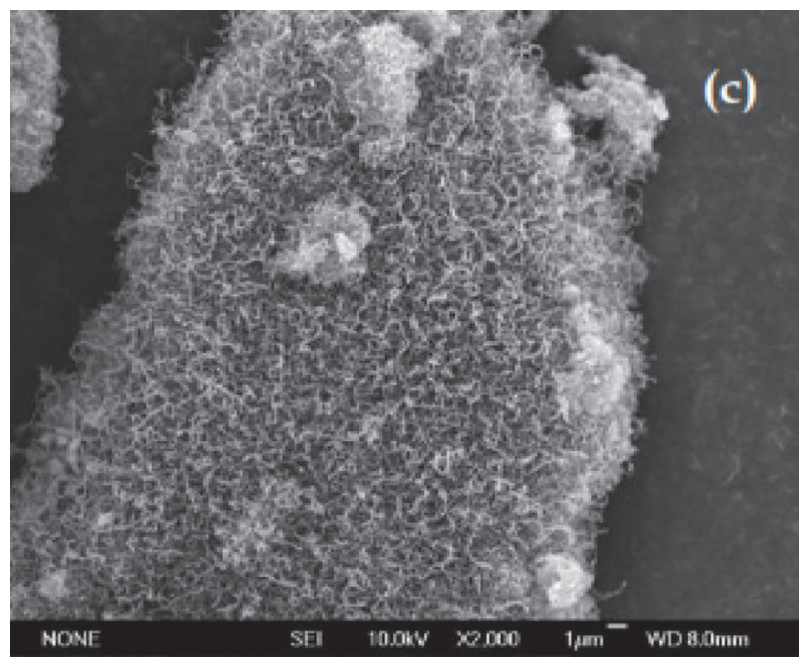

(c)

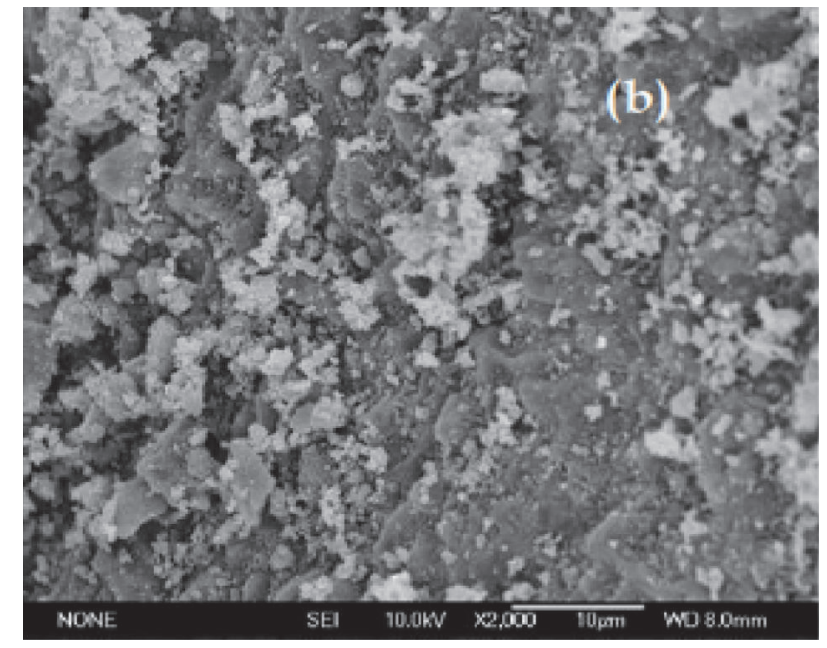

(b)

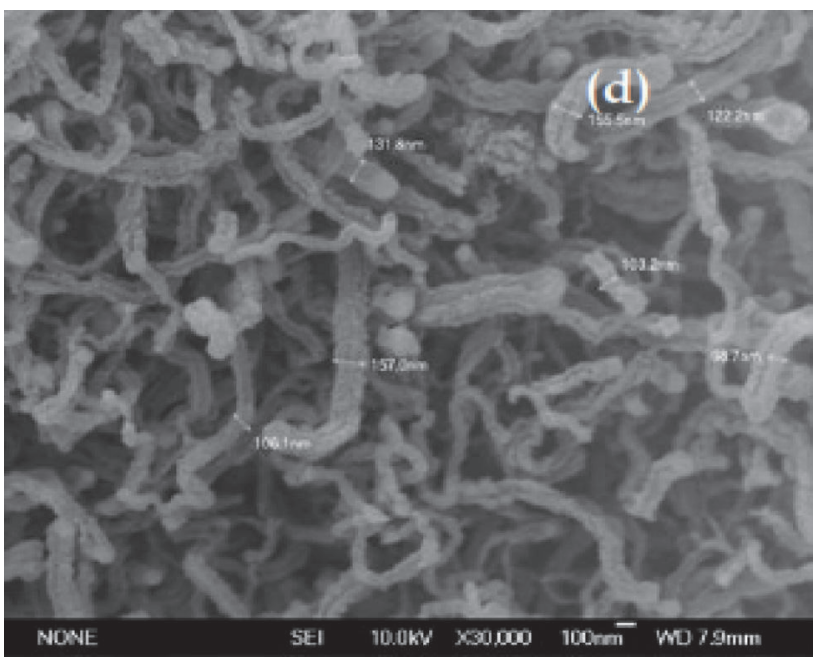

(d)

Figure 3: Scanning Electron Microscope images: (a) raw PAC, (b) Ni impregnated PAC, and (c) (d) CNF grown on PAC at optimized conditions.

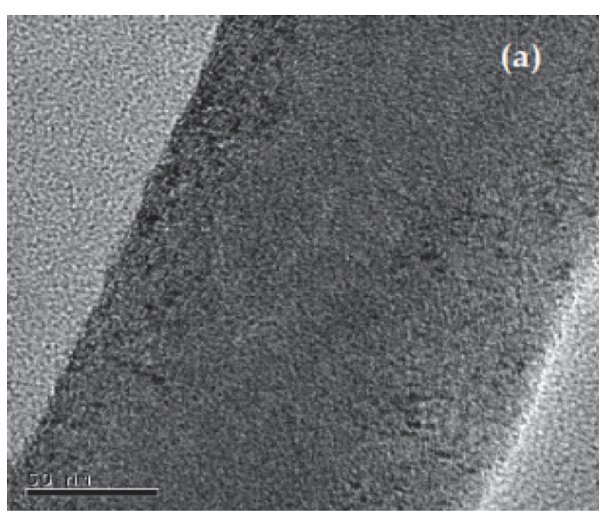

(a)

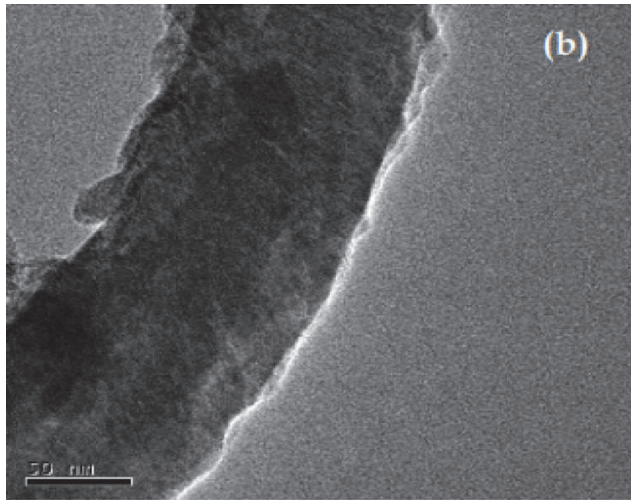

(b)

Figure 4: Transmission Electron Microscopy images CNF. 


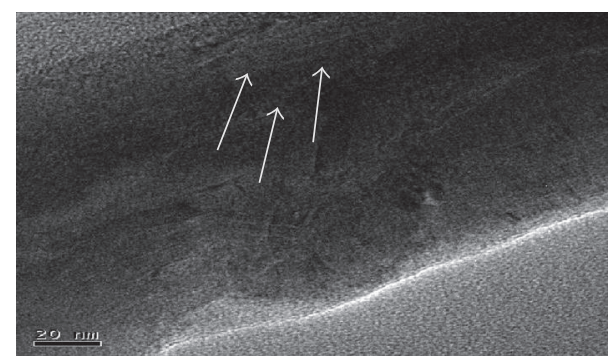

Figure 5: Transmission Electron Microscopy images of layered structure of CNF.

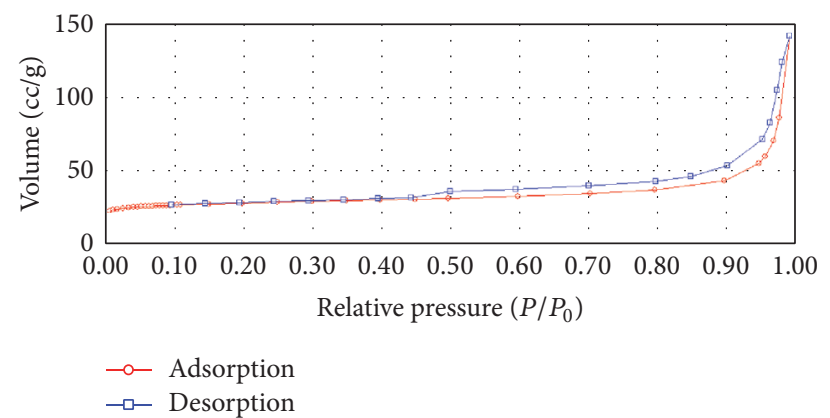

FIGURE 6: Adsorption/desorption of nitrogen gas on PAC.

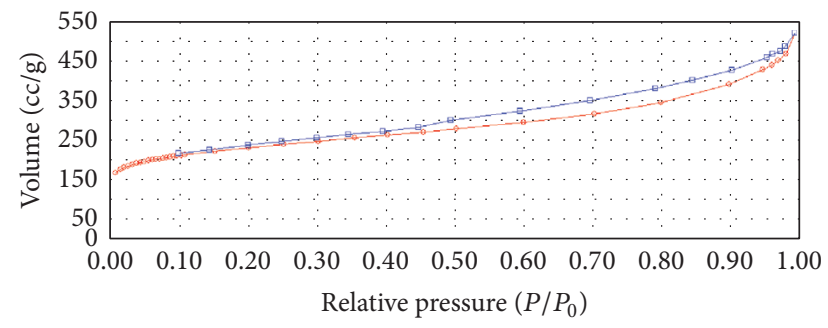

FIgURE 7: Adsorption/desorption of nitrogen gas on PAC-CNF.

were determined. The specific BET surface area of the PAC was found to be $101.1 \mathrm{~m}^{2} / \mathrm{g}$. After sonication, the $\mathrm{Ni}^{2+}$ impregnated PAC surface area increased to $131.2 \mathrm{~m}^{2} / \mathrm{g}$ due to production of additional fine particles during the sonication process. On the other hand, the CNF grown on PAC exhibited $836.7 \mathrm{~m}^{2} / \mathrm{g}$, which was more than eight times surface area compared to that of PAC.

Plot of volume of nitrogen gas adsorbed on raw PAC and PAC-CNF versus $P / P_{0}$ are shown in Figures 6 and 7 , respectively. The plot yields a line of slope $\left[(c-l) / c V_{m}\right]$ and intercept $\left[l / c V_{m}\right]$, where $V$ is the amount of the adsorbate. The values of $c, R^{2}$, and $V_{m}$ (monolayer capacity) were determined from the plots and the results are given in Table 2. As shown in Table 2, the new product, PAC-CNF, exhibited a higher monolayer capacity $\left(V_{m}\right)$ and total pore volume compared with the PAC, and this is in conformity with earlier reports $[39,40]$. These results are due to the increase in the surface area after the growth of the CNF on the PAC surface.
TABLE 2: BET isotherm parameters for nitrogen gas adsorption by PAC and PAC-CNF.

\begin{tabular}{lccc}
\hline \multirow{2}{*}{ Compound } & \multicolumn{3}{c}{ Parameter } \\
& Total pore volume $(\mathrm{cc} / \mathrm{g})$ & $V_{m}$ & $R^{2}$ \\
\hline PAC & $8.8 \times 10^{-2}$ & 0.029 & 0.9999 \\
Impregnated PAC & $3.26 \times 10^{-1}$ & 0.029 & 0.9996 \\
PAC-CNF & $6.75 \times 10^{-1}$ & 0.24 & 0.9985 \\
\hline
\end{tabular}

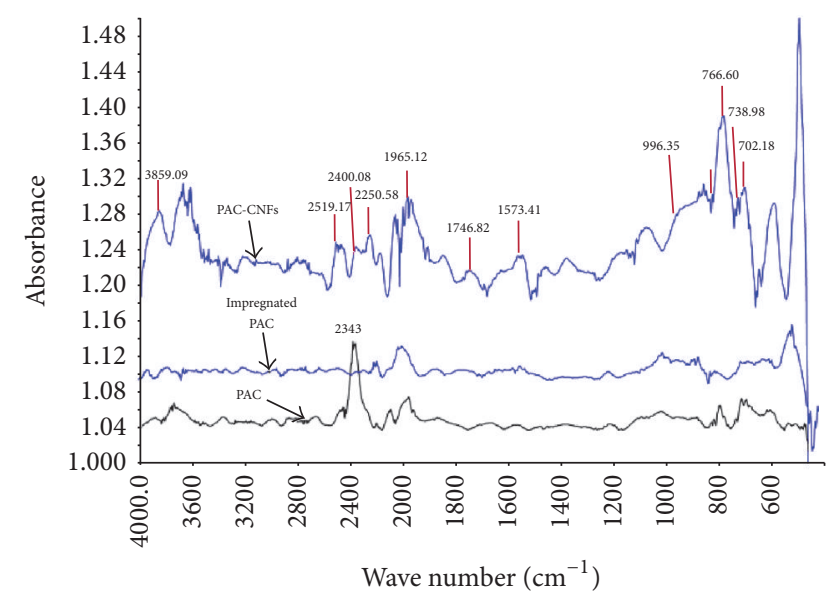

FIGURE 8: FTIR diagram of PAC, impregnated PAC, and PAC-CNF.

3.5. Zeta Potential. The zeta potential of the materials was measured for PAC and PAC-CNF in water. Test results showed that the zeta potential for the samples was negative and the peaks shifted towards zero point from PAC value $(-30.9)$ to the PAC-CNF value $(-24.9)$. The negative sign for the zeta potentials was indicative of the hydrophilicity of the composite. The high negative charge is an indication that the adsorbent is more hydrophilic [41]. The values of the zeta potential for the PAC after the growth of the CNF were still in the range of "incipient instability," a description similar to what was previously reported [42].

3.6. Surface Chemistry. The spectrum for the PAC, impregnated PAC, and CNF samples displayed bands with their respective functional groups as shown in Figure 8. The intensity of the band in the $3859-2519 \mathrm{~cm}^{-1}$ region contains $-\mathrm{OH}$ groups. The width and asymmetry of this band indicated the existence of strong hydrogen bonds, which could be due to adsorbed water on the PAC surfaces. The bands at 1965 and 1746 and $2250-2400 \mathrm{~cm}^{-1}$ were indicative of the presence of carbonyl groups $[43,44]$. Those bands at $996 \mathrm{~cm}^{-1}$ contain saturated carbon (methyl group $-\mathrm{CH}_{3}$ ) which might be from the cracking of acetylene [45].

The presence of these bands shows the formation of new carbon groups on the surface of PAC which is due to CNF [46]. Absorption peaks at 766 and $702 \mathrm{~cm}^{-1}$ represent $\mathrm{NO}_{2}$ bending vibrations [47]. These results were in agreement with the surface chemistry of other agricultural by-products $[45,48,49]$. Table 3 explains the peaks and functional groups represented by the bands. 
TABLE 3: Functional groups detected by FTIR on PAC, impregnated PAC, and PAC-CNF.

\begin{tabular}{lcc}
\hline Wave number at peak $\left(\mathrm{cm}^{-1}\right)$ & Functional group & Type of vibration \\
\hline $3859-2519$ & $-\mathrm{OH}$ (un-bonded), $-\mathrm{OH}$ (bonded) & No interferences such as $\mathrm{OH}$ in lattice, $-\mathrm{OH}$ stretch \\
$2400-2250 \& 1965,1746$ & Aldehyde, ketone, carboxylic acid & $\mathrm{C}=\mathrm{O}$, carbonyl group \\
2343 & $\mathrm{CO}_{2}$ & $\mathrm{C}=\mathrm{C}$ stretch \\
1573 & $\mathrm{C}=\mathrm{C}$ aromatic ring & Saturated $-\mathrm{CH}_{3}$ \\
996 & Methyl group & Bending \\
$766 \& 702$ & $\mathrm{NO}_{2}$ & Adjacent hydrogens on an aromatic ring (Ortho) \\
739 & Hydrogen on an aromatic ring &
\end{tabular}

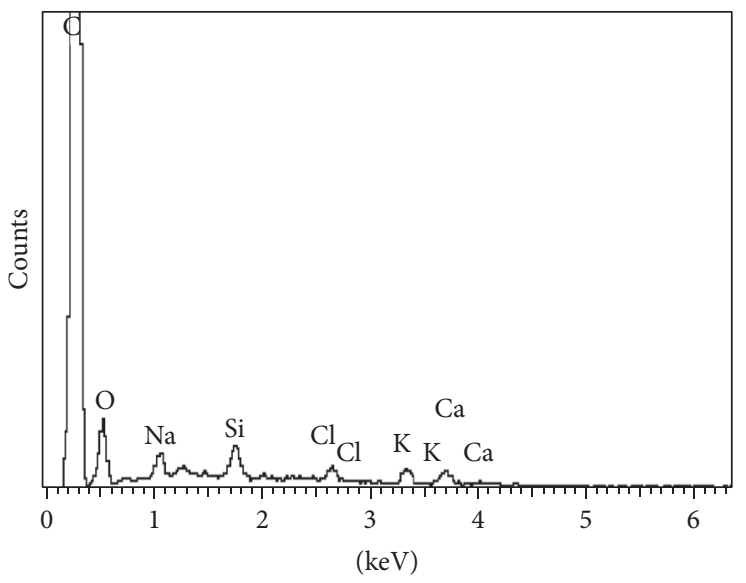

(a)

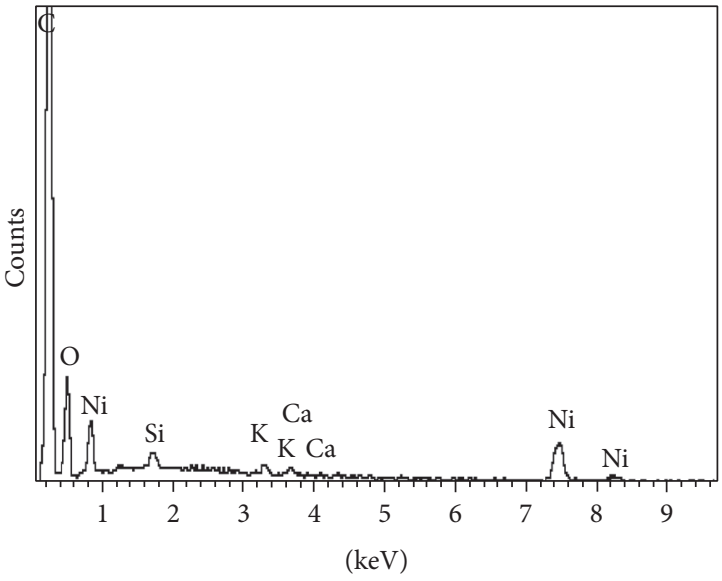

(b)

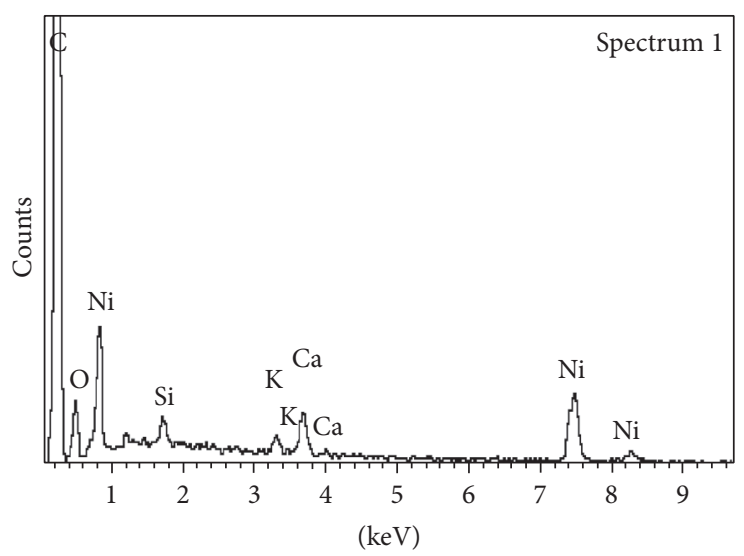

(c)

FIgure 9: EDX spectrums for (a) PAC, (b) Ni impregnated PAC, and (c) PAC-CNF.

3.7. Energy Dispersive X-Ray (EDX) Analysis. The EDX imaging analysis revealed the materials' surface structure and the local elements distribution of PAC, impregnated PAC, and PAC-CNF were examined using the EDX analyzer as depicted in Figure 9 [50]. Figures 9(a), 9(b), and 9(c) showed the electron images for the samples; this invariably showed the difference in the surface structure between the four compounds. These observations conformed to the spectrum images shown in Figure 9.

It was observed in Figure 9 that carbon element was present in high percentage in the four mentioned samples followed by oxygen and other elements as impurities ( $\mathrm{Na}$,
$\mathrm{Si}, \mathrm{Cl}, \mathrm{K}$, and $\mathrm{Ca}$ ) with low percentages. The existence of nickel element on the surface of the PAC after impregnation of the element was also established, and this proved the success of the impregnation process. The elements detected by the EDX analyzer with their respective average weight and atomic percentages are presented in Table 4 . The table showed that the percentage of the atomic carbon increased from $87 \%$ to $94 \%$ after the CNF growth on the surface of the substrate which enhances the decomposition of the acetylene to atomic carbon, similar to what was reported previously [51]. The presence of nickel on the surface of PAC was about $6.2 \%$. 
TABle 4: Compositions of elements in PAC, Ni impregnated PAC, and PAC-CNF.

\begin{tabular}{|c|c|c|c|c|c|c|}
\hline \multirow{2}{*}{ Element } & \multicolumn{3}{|c|}{ Average weight (\%) } & \multicolumn{3}{|c|}{ Average atomic composition (\%) } \\
\hline & PAC & Ni-PAC & PAC-CNF & $\mathrm{PAC}$ & Ni-PAC & $\mathrm{PAC}-\mathrm{CNH}$ \\
\hline $\mathrm{C}$ & 89.98 & 78.85 & 87.37 & 92.10 & 86.69 & 94.05 \\
\hline $\mathrm{O}$ & 4.88 & 13.96 & 3.95 & 7.20 & 11.52 & 6.66 \\
\hline $\mathrm{Na}$ & 0.18 & - & - & 0.10 & - & - \\
\hline $\mathrm{Si}$ & 0.71 & 0.37 & 0.69 & 0.32 & 0.18 & 0.32 \\
\hline $\mathrm{Cl}$ & 0.32 & - & - & 0.40 & - & - \\
\hline $\mathrm{K}$ & 0.33 & 0.36 & 0.55 & 0.11 & 0.12 & 0.18 \\
\hline $\mathrm{Ca}$ & 0.43 & 0.26 & 0.31 & 0.13 & 0.08 & 0.22 \\
\hline $\mathrm{Ni}$ & - & 6.16 & 5.84 & - & 1.39 & 1.29 \\
\hline
\end{tabular}

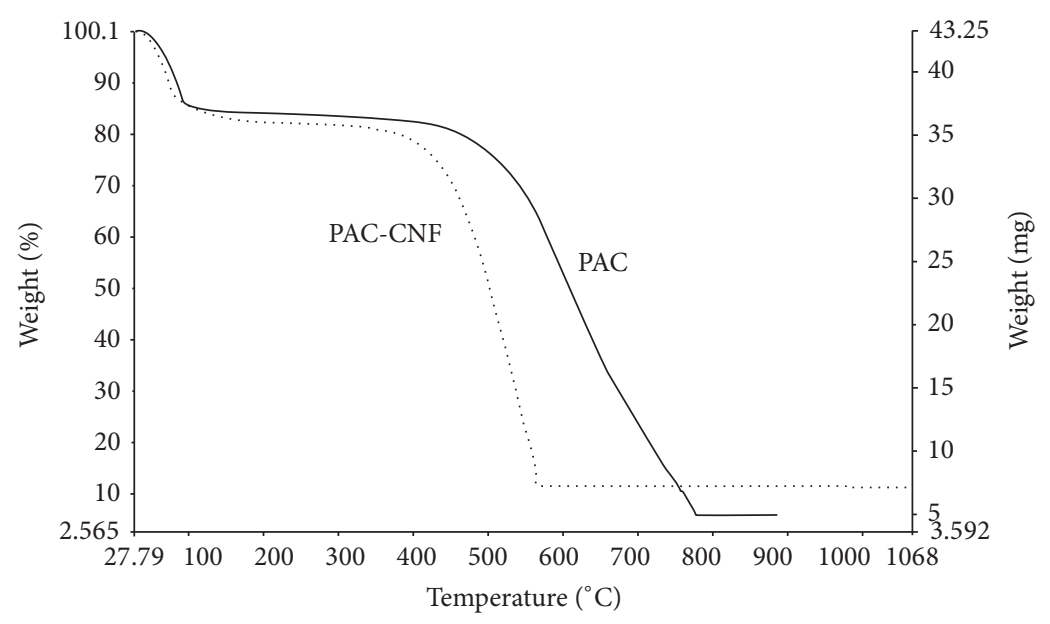

FIgURe 10: Thermal stability of PAC and PAC-CNF.

3.8. Thermogravimetric Analysis (TGA). Thermogravimetric analysis (TGA) revealed the temperature effect on the PAC and CNF. The TGA profiles obtained under the inert gas condition are presented in Figure 10. The first weight loss occurring around $100^{\circ} \mathrm{C}$ of temperature was observed for the samples which was caused by thermodesorption of physically adsorbed material such as water vapor (moisture) and hydrocarbons [52] and it showed that the moisture content was around $15-18 \%$ for PAC and CNF, respectively.

High temperatures (between 400 and $480^{\circ} \mathrm{C}$ ) for $\mathrm{CNF}$ and $\mathrm{PAC}$, respectively, led to the start of combustion. Decrease in weight is commonly considered to be due to the vaporization of volatile organic compounds [53], which was indicated by a significant weight loss of $70 \% \mathrm{w} / \mathrm{w}$. It was also observed that the weight loss stopped after a higher limit of combustion temperature (570 and $780^{\circ} \mathrm{C}$ for $\mathrm{CNF}$ and PAC, resp.) was reached. Some residual material remained for all samples, and these residues indicated the carbon purity of the samples with PAC having 5\% representing the ash content and PACCNF exhibiting $8 \%$, which was due to the $\mathrm{Ni}^{2+}$ catalyst impregnated on PAC surface prior to the CNF growth.

\section{Conclusions}

The morphological property of the oil palm kernel shellbased PAC was modified through the impregnation of $\mathrm{Ni}$ catalyst, followed by the synthesis of CNF with the presence of acetylene as a carbon source. Dispersion of $\mathrm{Ni}^{2+}$ catalyst was successfully carried out with the use of acetone sonication for nickel (II) nitrate hexahydrate to arrive at the successful growth of CNF. Several concentrations of $\mathrm{Ni}^{2+}$ catalysts were tested and the best growth of CNF was achieved at $3 \% \mathrm{Ni}^{2+}$ (w/w). The FESEM and TEM showed the graphitic structure of the PAC-CNF, BET surface area $836.7 \mathrm{~m}^{2} / \mathrm{g}$, zeta potential $-24.9 \mathrm{mV}$, and TGA combustion temperature range between $400^{\circ} \mathrm{C}$ and $570^{\circ} \mathrm{C}$.

\section{Competing Interests}

The authors declare that they have no competing interests.

\section{Acknowledgments}

The authors are grateful to the Ministry of Higher Education (MOHE), Government of Malaysia, for funding this project by the Fundamental Research Grant Scheme with Grant no. FRGS 0106-42 administrated by the ministry (MOHE). The authors duly appreciate the assistance rendered by Sajid and Imtiaz in typing and formatting the manuscript. Assistance from the laboratory technicians of IIUM and University Malaya (UM) is also acknowledged. 


\section{References}

[1] H. Marsh and F. Rodríguez-Reinoso, Activated Carbon, Elsevier, Oxford, UK, 2006.

[2] Y. Han, X. Quan, H. Zhao, S. Chen, and Y. Zhao, "Kinetics of enhanced adsorption by polarization for organic pollutants on activated carbon fiber," Frontiers of Environmental Science and Engineering in China, vol. 1, no. 1, pp. 83-88, 2007.

[3] G. Issabayeva, M. K. Aroua, and N. M. N. Sulaiman, "Removal of lead from aqueous solutions on palm shell activated carbon," Bioresource Technology, vol. 97, no. 18, pp. 2350-2355, 2006.

[4] D. Mohan and C. U. Pittman Jr., "Activated carbons and low cost adsorbents for remediation of tri- and hexavalent chromium from water," Journal of Hazardous Materials, vol. 137, no. 2, pp. 762-811, 2006.

[5] C. K. Ahn, D. Park, S. H. Woo, and J. M. Park, "Removal of cationic heavy metal from aqueous solution by activated carbon impregnated with anionic surfactants," Journal of Hazardous Materials, vol. 164, no. 2-3, pp. 1130-1136, 2009.

[6] M. Gurrath, T. Kuretzky, H. P. Boehm, L. B. Okhlopkova, A. S. Lisitsyn, and V. A. Likholobov, "Palladium catalysts on activated carbon supports Influence of reduction temperature, origin of the support and pretreatments of the carbon surface," Carbon, vol. 38, no. 8, pp. 1241-1255, 2000.

[7] H. Jüntgen, "Activated carbon as catalyst support: a review of new research results," Fuel, vol. 65, no. 10, pp. 1436-1446, 1986.

[8] M. R. Cuervo, E. Asedegbega-Nieto, E. Díaz et al., "Effect of carbon nanofiber functionalization on the adsorption properties of volatile organic compounds," Journal of Chromatography A, vol. 1188, no. 2, pp. 264-273, 2008.

[9] H. Song and W. Shen, "Carbon nanofibers: synthesis and applications," Journal of Nanoscience and Nanotechnology, vol. 14, no. 2, pp. 1799-1810, 2014.

[10] E. Hammel, X. Tang, M. Trampert et al., "Carbon nanofibers for composite applications," Carbon, vol. 42, no. 5-6, pp. 1153-1158, 2004.

[11] E. Díaz, S. Ordóñez, and A. Vega, "Adsorption of volatile organic compounds onto carbon nanotubes, carbon nanofibers, and high-surface-area graphites," Journal of Colloid and Interface Science, vol. 305, no. 1, pp. 7-16, 2007.

[12] A. Chatterjee and B. L. Deopura, "Carbon nanotubes and nanofibre: an overview," Fibers and Polymers, vol. 3, no. 4, pp. 134-139, 2002.

[13] M. Inagaki and L. R. Radovic, "Nanocarbons," Carbon, vol. 40, no. 12, pp. 2279-2282, 2002.

[14] M. S. P. Shaffer and J. K. W. Sandler, Carbon Nanotube/Nanofiber Composites, 2008.

[15] M. Pacheco, J. Pacheco, and R. Valdivia, "Synthesis of Carbon Nanofibers by a Glow-arc Discharge," 2010.

[16] Y. Suda, A. Tanaka, A. Okita, Y. Sakai, and H. Sugawara, "Growth of carbon nanofibers on metal-catalyzed substrates by pulsed laser ablation of graphite," Journal of Physics: Conference Series, vol. 59, no. 1, pp. 348-353, 2007.

[17] I. Martin-Gullon, J. Vera, J. A. Conesa, J. L. González, and C. Merino, "Differences between carbon nanofibers produced using $\mathrm{Fe}$ and Ni catalysts in a floating catalyst reactor," Carbon, vol. 44, no. 8, pp. 1572-1580, 2006.

[18] H. Huang, H. Kajiura, Y. Murakami, and M. Ata, "Metal sulfide catalyzed growth of carbon nanofibers and nanotubes," Carbon, vol. 41, no. 3, pp. 615-618, 2003.
[19] H. Kajiura, H. Huang, S. Tsutsui, Y. Murakami, and M. Miyakoshi, "High-purity fibrous carbon deposit on the anode surface in hydrogen DC arc-discharge," Carbon, vol. 40, no. 13, pp. 2423-2428, 2002.

[20] C. J. Lee, T. J. Lee, and J. Park, "Carbon nanofibers grown on sodalime glass at $500 \circ \mathrm{C}$ using thermal chemical vapor deposition," Chemical Physics Letters, vol. 340, no. 5-6, pp. 413$418,2001$.

[21] H. O. Pierson, Handbook of Chemical Vapor Deposition: Principles, Technology and Applications, William Andrew, New York, NY, USA, 2nd edition, 1999.

[22] L. Ci, J. Wei, B. Wei, J. Liang, C. Xu, and D. Wu, "Carbon nanofibers and single-walled carbon nanotubes prepared by the floating catalyst method," Carbon, vol. 39, no. 3, pp. 329-335, 2001.

[23] C. Vallés, M. Pérez-Mendoza, W. K. Maser et al., "Effects of partial and total methane flows on the yield and structural characteristics of MWCNTs produced by CVD," Carbon, vol. 47, no. 4, pp. 998-1004, 2009.

[24] W. Z. Li, J. G. Wen, and Z. F. Ren, "Effect of temperature on growth and structure of carbon nanotubes by chemical vapor deposition," Applied Physics A, vol. 74, no. 3, pp. 397-402, 2002.

[25] C. Pham-Huu, R. Vieira, B. Louis et al., "About the octopuslike growth mechanism of carbon nanofibers over graphite supported nickel catalyst," Journal of Catalysis, vol. 240, no. 2, pp. 194-202, 2006.

[26] G. J. Dahe, R. S. Teotia, and J. R. Bellare, "The role of zeolite nanoparticles additive on morphology, mechanical properties and performance of polysulfone hollow fiber membranes," Chemical Engineering Journal, vol. 197, pp. 398-406, 2012.

[27] A. Romero, A. Garrido, A. Nieto-Márquez, A. R. de la Osa, A. de Lucas, and J. L. Valverde, "The influence of operating conditions on the growth of carbon nanofibers on carbon nanofibersupported nickel catalysts," Applied Catalysis A: General, vol. 319, pp. 246-258, 2007.

[28] M. C. Díaz, J. M. Blackman, and C. E. Snape, "Maximising carbon nanofiber and hydrogen production in the catalytic decomposition of ethylene over an unsupported $\mathrm{Ni}-\mathrm{Cu}$ alloy," Applied Catalysis A: General, vol. 339, no. 2, pp. 196-208, 2008.

[29] S. Esconjauregui, C. M. Whelan, and K. Maex, "The reasons why metals catalyze the nucleation and growth of carbon nanotubes and other carbon nanomorphologies," Carbon, vol. 47, no. 3, pp. 659-669, 2009.

[30] N. K. Reddy, J.-L. Meunier, and S. Coulombe, "Growth of carbon nanotubes directly on a nickel surface by thermal CVD," Materials Letters, vol. 60, no. 29-30, pp. 3761-3765, 2006.

[31] A. E. Aksoylu, J. L. Faria, M. F. R. Pereira et al., "Highly dispersed activated carbon supported platinum catalysts prepared by OMCVD: a comparison with wet impregnated catalysts," Applied Catalysis A: General, vol. 243, no. 2, pp. 357-365, 2003.

[32] M.-J. Ledoux and C. Pham-Huu, "Carbon nanostructures with macroscopic shaping for catalytic applications," Catalysis Today, vol. 102-103, pp. 2-14, 2005.

[33] C.-H. Wu, J.-F. Shr, C.-F. Wu, and C.-T. Hsieh, "Adsorption energy distribution of carbon tetrachloride on carbon nanofiber arrays prepared by template synthesis," Physica E: Low-Dimensional Systems and Nanostructures, vol. 40, no. 4, pp. 814-821, 2008.

[34] Y.-Y. Fan, H.-M. Cheng, Y.-L. Wei, G. Su, and Z.-H. Shen, "Tailoring the diameters of vapor-grown carbon nanofibers," Carbon, vol. 38, no. 6, pp. 921-927, 2000. 
[35] Y.-Y. Fan, H.-M. Cheng, Y.-L. Wei, G. Su, and Z.-H. Shen, “The influence of preparation parameters on the mass production of vapor-grown carbon nanofibers," Carbon, vol. 38, no. 6, pp. 789795, 2000.

[36] R. L. Vander Wal, T. M. Ticich, and V. E. Curtis, "Directed synthesis of metal-catalyzed carbon nanofibers and graphite encapsulated metal nanoparticles," The Journal of Physical Chemistry B, vol. 104, no. 49, pp. 11606-11611, 2000.

[37] P. Tribolet and L. Kiwi-Minsker, "Carbon nanofibers grown on metallic filters as novel catalytic materials," Catalysis Today, vol. 102-103, pp. 15-22, 2005.

[38] K. Aoki, T. Yamamoto, H. Furuta et al., "Low-temperature growth of carbon nanofiber by thermal chemical vapor deposition using CuNi catalyst," Japanese Journal of Applied Physics, vol. 45, no. 6, pp. 5329-5331, 2006.

[39] R. A. Khalkhali and R. Omidvari, "Adsorption of mercuric ion from aqueous solutions using activated carbon," Polish Journal of Environmental Studies, vol. 14, no. 2, pp. 185-188, 2005.

[40] K. Byrappa, A. K. Subramani, S. Ananda et al., "Impregnation of $\mathrm{ZnO}$ onto activated carbon under hydrothermal conditions and its photocatalytic properties," Journal of Materials Science, vol. 41, no. 5, pp. 1355-1362, 2006.

[41] W. Omar and H. Al-Itawi, "Removal of $\mathrm{Pb}^{+2}$ ions from aqueous solutions by adsorption on kaolinite clay," American Journal of Applied Sciences, vol. 4, no. 7, pp. 502-507, 2007.

[42] E. Ofir, Y. Oren, and A. Adin, "Electroflocculation: the effect of zeta-potential on particle size," Desalination, vol. 204, no. 1-3, pp. 33-38, 2007.

[43] Y.-M. Li, S.-Q. Sun, Q. Zhou et al., "Identification of American ginseng from different regions using FT-IR and twodimensional correlation IR spectroscopy," Vibrational Spectroscopy, vol. 36, no. 2, pp. 227-232, 2004.

[44] R. Nadeem, T. M. Ansari, K. Akhtar, and A. M. Khalid, "Pb(II) sorption by pyrolysed Pongamia pinnata pods carbon (PPPC)," Chemical Engineering Journal, vol. 152, no. 1, pp. 54-63, 2009.

[45] C. Bouchelta, M. S. Medjram, O. Bertrand, and J.-P. Bellat, "Preparation and characterization of activated carbon from date stones by physical activation with steam," Journal of Analytical and Applied Pyrolysis, vol. 82, no. 1, pp. 70-77, 2008.

[46] Y. Ominami, Q. Ngo, A. J. Austin et al., "Structural characteristics of carbon nanofibers for on-chip interconnect applications," Applied Physics Letters, vol. 87, no. 33, p. 233105, 2005.

[47] A.-N. A. El-Hendawy, "Variation in the FTIR spectra of a biomass under impregnation, carbonization and oxidation conditions," Journal of Analytical and Applied Pyrolysis, vol. 75, no. 2, pp. 159-166, 2006.

[48] Y. Al-Degs, M. A. M. Khraisheh, S. J. Allen, and M. N. Ahmad, "Effect of carbon surface chemistry on the removal of reactive dyes from textile effluent," Water Research, vol. 34, no. 3, pp. 927-935, 2000.

[49] C. Sellitti, J. L. Koenig, and H. Ishida, "Surface characterization of graphitized carbon fibers by attenuated total reflection fourier transform infrared spectroscopy," Carbon, vol. 28, no. 1, pp. 221-228, 1990.

[50] M. Schulze, M. V. Bradke, R. Reissner, M. Lorenz, and E. Gülzow, "Characterization of polymers in PEFC-electrodes with EDX and XPS," Fresenius' Journal of Analytical Chemistry, vol. 365, no. 1-3, pp. 123-132, 1999.

[51] C.-C. Chuang, W.-L. Liu, W.-J. Chen, and J.-H. Huang, “Temperature and substrate dependence of structure and growth mechanism of carbon nanofiber," Applied Surface Science, vol. 254, no. 15, pp. 4681-4687, 2008.
[52] M. M. Maroto-Valer, I. Dranca, T. Lupascu, and R. Nastas, "Effect of adsorbate polarity on thermodesorption profiles from oxidized and metal-impregnated activated carbons," Carbon, vol. 42, no. 12-13, pp. 2655-2659, 2004.

[53] J.-W. Kim, M.-H. Sohn, D.-S. Kim, S.-M. Sohn, and Y.-S. Kwon, "Production of granular activated carbon from waste walnut shell and its adsorption characteristics for $\mathrm{Cu}^{2+}$ ion," Journal of Hazardous Materials, vol. 85, no. 3, pp. 301-315, 2001. 

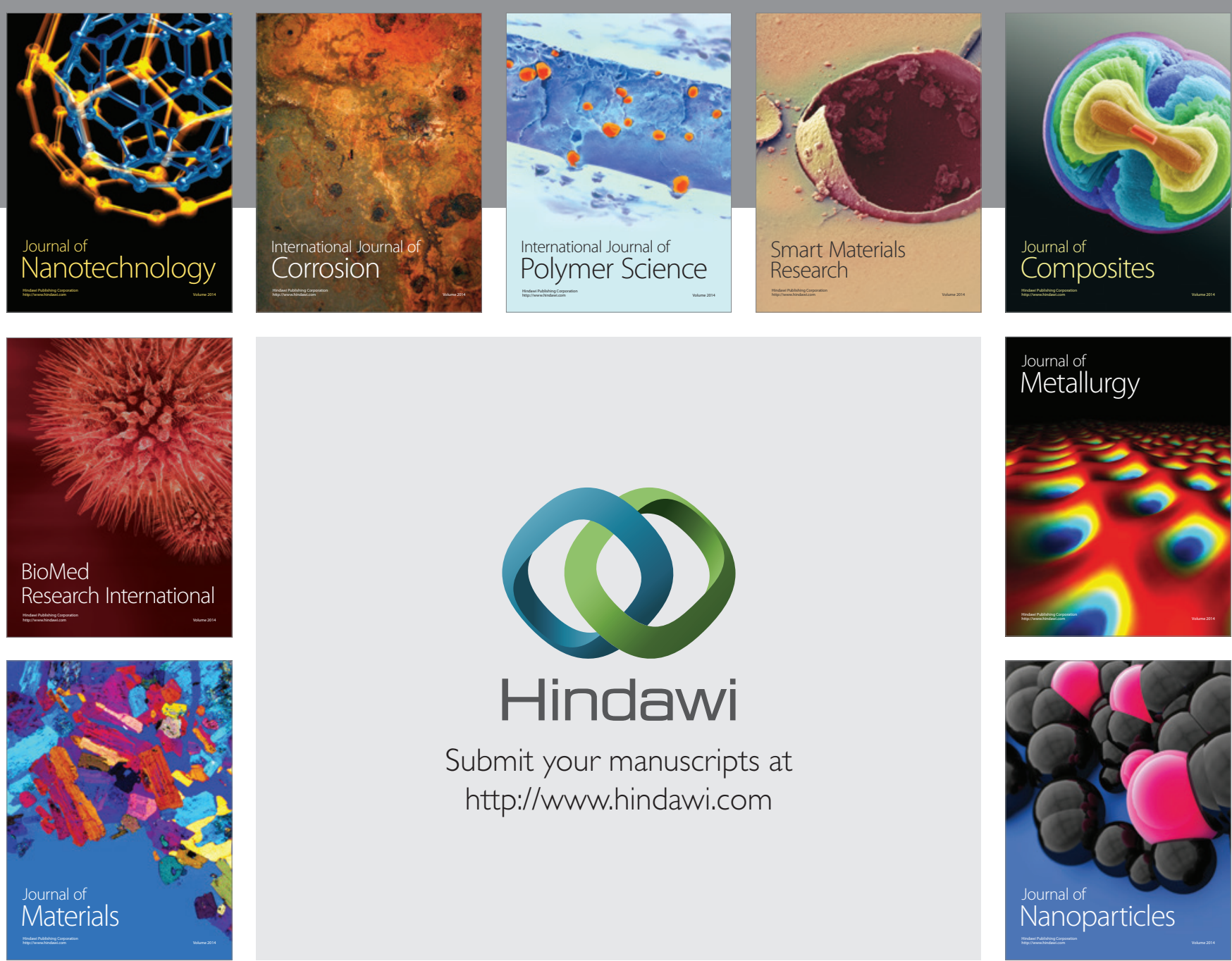

\section{Hindawi}

Submit your manuscripts at

http://www.hindawi.com

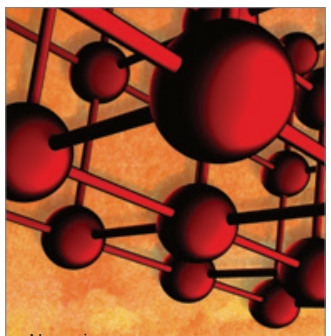

Materials Science and Engineering
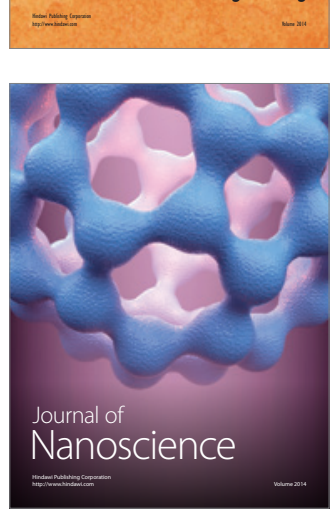
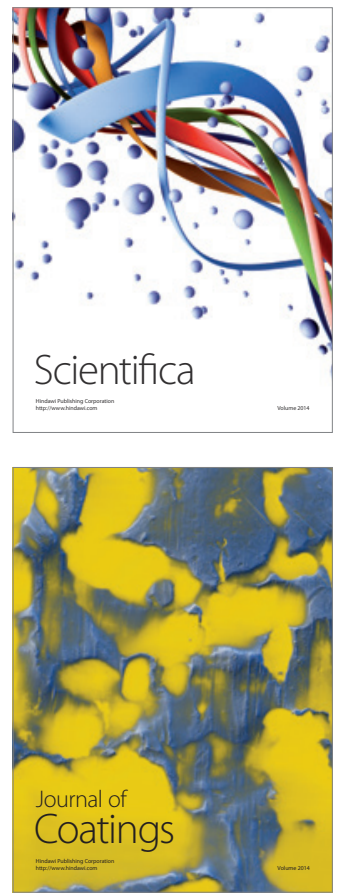
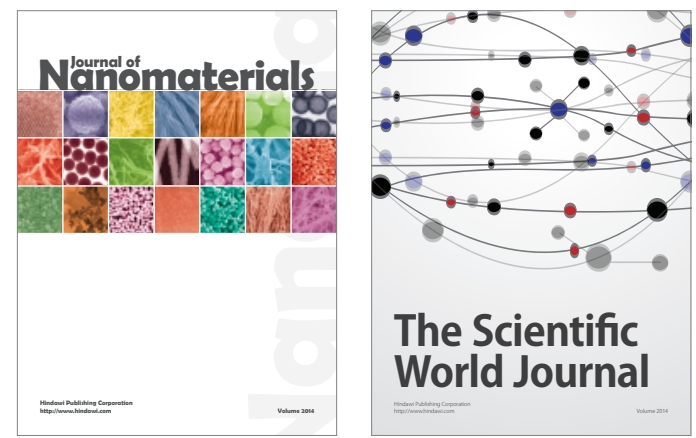

The Scientific World Journal
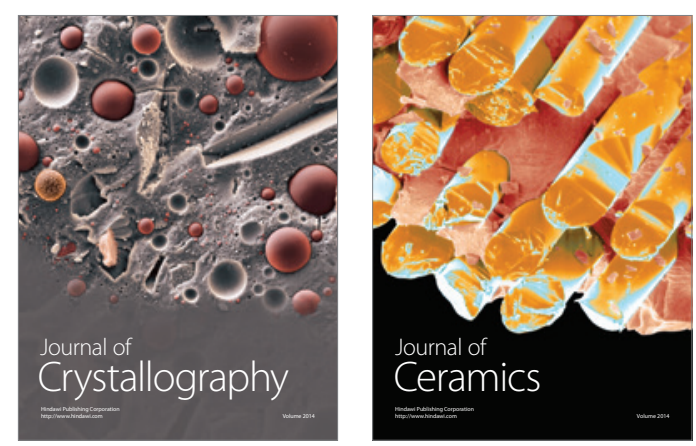
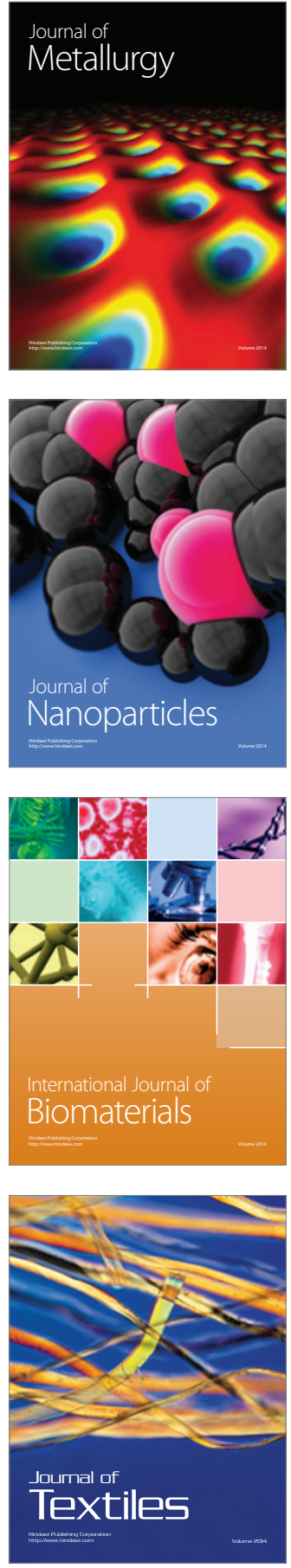